\title{
Front Matter: Volume 10677
}

, "Front Matter: Volume 10677," Proc. SPIE 10677, Unconventional Optical Imaging, 1067701 (13 August 2018); doi: 10.1117/12.2503289

SPIE. Event: SPIE Photonics Europe, 2018, Strasbourg, France 


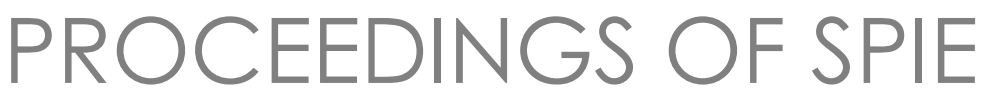

\title{
Unconventional Optical Imaging
}

\author{
Corinne Fournier \\ Marc P. Georges \\ Gabriel Popescu \\ Editors
}

22-26 April 2018

Strasbourg, France

Sponsored by

SPIE

Cosponsored by

Strasbourg the Europtimist (France)

CNRS (France)

Investissements d'Avenvir (France)

iCube (France)

Université de Strasbourg (France)

Cooperating Organisations

Photonics 21 (Germany)

EOS-European Optical Society (Germany)

Photonics Public Private Partnership (Belgium)

Comité National d'Optique et de Photonique (France)

Published by

SPIE 
The papers in this volume were part of the technical conference cited on the cover and title page. Papers were selected and subject to review by the editors and conference program committee. Some conference presentations may not be available for publication. Additional papers and presentation recordings may be available online in the SPIE Digital Library at SPIEDigitallibrary.org.

The papers reflect the work and thoughts of the authors and are published herein as submitted. The publisher is not responsible for the validity of the information or for any outcomes resulting from reliance thereon.

Please use the following format to cite material from these proceedings:

Author(s), "Title of Paper," in Unconventional Optical Imaging, edited by Corinne Fournier, Marc P. Georges, Gabriel Popescu, Proceedings of SPIE Vol. 10677 (SPIE, Bellingham, WA, 2018) Seven-digit Article CID Number.

ISSN: 0277-786X

ISSN: 1996-756X (electronic)

ISBN: 9781510618800

ISBN: 9781510618817 (electronic)

Published by

SPIE

P.O. Box 10, Bellingham, Washington 98227-0010 USA

Telephone +1 3606763290 (Pacific Time) · Fax +1 3606471445

SPIE.org

Copyright @ 2018 , Society of Photo-Optical Instrumentation Engineers.

Copying of material in this book for internal or personal use, or for the internal or personal use of specific clients, beyond the fair use provisions granted by the U.S. Copyright Law is authorized by SPIE subject to payment of copying fees. The Transactional Reporting Service base fee for this volume is $\$ 18.00$ per article (or portion thereof), which should be paid directly to the Copyright Clearance Center (CCC), 222 Rosewood Drive, Danvers, MA 01923. Payment may also be made electronically through CCC Online at copyright.com. Other copying for republication, resale, advertising or promotion, or any form of systematic or multiple reproduction of any material in this book is prohibited except with permission in writing from the publisher. The CCC fee code is 0277$786 \mathrm{X} / 18 / \$ 18.00$.

Printed in the United States of America.

Publication of record for individual papers is online in the SPIE Digital Library.

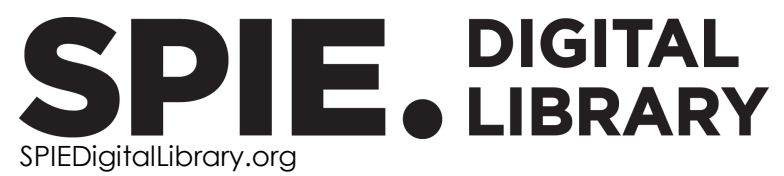

Paper Numbering: Proceedings of SPIE follow an e-First publication model. A unique citation identifier (CID) number is assigned to each article at the time of publication. Utilization of CIDs allows articles to be fully citable as soon as they are published online, and connects the same identifier to all online and print versions of the publication. SPIE uses a seven-digit CID article numbering system structured as follows:

- The first five digits correspond to the SPIE volume number.

- The last two digits indicate publication order within the volume using a Base 36 numbering system employing both numerals and letters. These two-number sets start with $00,01,02,03,04$, 05, 06, 07, 08, 09, 0A, OB ... 0Z, followed by 10-1Z, 20-2Z, etc. The CID Number appears on each page of the manuscript. 


\title{
Contents
}

\author{
xi Authors \\ xv Conference Committee
}

MODELLING, COMPUTATION AND DESIGN I: CO-DESIGN FOR UNCONVENTIONAL IMAGING

1067703 Increasing image resolution in near-infrared to visible upconversion detection for long-range active imaging [10677-2]

1067704 Color correction matrix for sparse RGB-W image sensor without IR cutoff filter [10677-3]

1067705 Embedded video rate super-resolution in the infrared with a low-cost multi-aperture camera [10677-4]

1067706 Simple and cheap hyperspectral imaging for astronomy (and more) [10677-5]

1067707 Experimental demonstration of diffraction-limited plenoptic imaging [10677-6]

MODELLING, COMPUTATION AND DESIGN II: CO-DESIGN FOR UNCONVENTIONAL IMAGING

1067709 Chromatic add-on to improve depth from defocus with a conventional camera [10677-8]

10677 OA SNR-optimized image fusion for transparent object inspection [10677-9]

10677 OB Pansharpening of images acquired with color filter arrays [10677-10]

10677 OD Time-resolved diffuse optical tomography system based on adaptive structured light illumination and compressive sensing detection [10677-13]

10677 OE Imaging VLS grating spectrographs [10677-14]

10677 OF Calibration of a stereoscopic video endoscope for precise three-dimensional geometrical measurements in arbitrary spectral bands [10677-15]

APPLICATIONS I: BIOIMAGING

$10677 \mathrm{OH} \quad$ Quantitative phase microscopy of dynamic cells using off-axis holographic compression by spatial multiplexing [10677-17] 
10677 Ol Color holographic microscope for monitoring lipids in microalgae [10677-18]

10677 OJ Tomographic diffractive microscopy: principles and applications (Keynote Paper) [10677-136]

APPLICATIONS II: BIOIMAGING

10677 OK Advances in optical coherence tomography for dermatology (Invited Paper) [10677-19]

10677 OM Quantitative phase retrieval reconstruction from in-line hologram using a new proximal operator: application to microscopy of bacteria and tracking of droplets [10677-21]

1067700 Bioimaging with controlled depth using upconversion nanoparticles [10677-23]

10677 OP Label-free imaging of lipid droplets in cells by stimulated Raman microscopy [10677-24]

APPLICATIONS III: MICRO- NANOSCOPIC IMAGING

10677 OR Through-focus scanning optical microscopy applications (Invited Paper) [10677-26]

10677 OV Laser sources in multiphoton microscopy: overview and optimization [10677-30]

MODELLING, COMPUTATION AND DESIGN III

10677 OW Partially coherent imaging in phase space (Invited Paper) [10677-31]

10677 OX Development of a realistic wave propagation-based chromatic confocal microscopy model [10677-32]

10677 OY A dimension reduction method for fast diffuse optical tomography [10677-33]

1067710 Super-resolution for noisy images via deep convolutional neural network [10677-35]

\section{ADVANCED METHODS I: LIGHT SCATTERING}

1067715 Vision through turbid media by Fourier filtering and single-pixel detection (Best Student Paper Award) [10677-40]

1067717 Scatter-plate microscope: improved image acquisition [10677-42]

iv 
1067718 Mueller microscopy of anisotropic scattering media: theory and experiments [10677-43]

ADVANCED METHODS II: QUANTITATIVE PHASE IMAGING

1067719 Off-axis digital holography for flow analysis (Invited Paper) [10677-44]

10677 1A Improving color lensless microscopy reconstructions by self-calibration [10677-45]

10677 1B Multiwavelength surface contouring from phase-coded diffraction patterns [10677-46]

10677 1C Quantitative phase imaging in turbid media by coherence controlled holographic microscopy [10677-47]

10677 IE Ptychography: quantitative phase imaging with incoherent imaging properties [10677-50]

$10677 \mathrm{IF} \quad$ Simplified approach for tomographic diffractive microscopy of axisymmetric samples [10677-48]

OPTICS IN SURGERY I: JOINT SESSION BETWEEN CONFERENCES 10677 AND 10685

$106771 G$ Phantom and methodology for fluorescence molecular imaging systems benchmarking [10677-52]

OPTICS IN SURGERY II: JOINT SESSION BETWEEN CONFERENCES 10677 AND 10685

$106771 \mathrm{~J} \quad$ Optics in surgery: the surgeon perspective (Invited Paper) [10677-54]

$10677 \mathrm{~K}$ Near-infrared fluorescence imaging methods to evaluate blood flow state in the skin lesions [10677-55]

ADVANCED METHODS III: QUANTITATIVE PHASE IMAGING

10677 IQ In-line and off-axis hybrid digital holography [10677-62]

10677 IR Quantitative phase imaging by using a position sensitive detector [10677-61] 


\section{APPLICATIONS V: WAVEFRONT SHAPING AND RESTORATION}

10677 is Phase diversity: math, methods and prospects, including sequential diversity imaging (Invited Paper) [10677-63]

10677 IW Interest of polarimetric refocused images calibrated in depth for control by vision [10677-68]

ADVANCED METHODS IV: TERAHERTZ IMAGING

$106771 \mathrm{X} \quad$ Continuous-wave terahertz phase-contrast imaging (Invited Paper) [10677-69]

10677 IY A potential of terahertz solid immersion microscopy for visualizing sub-wavelength-scale tissue spheroids [10677-70]

$1067712 \quad$ Terahertz pulsed imaging reveals the stratigraphy of a seventeenth-century oil painting [10677-71]

1067720 Resolution limits of terahertz ptychography (Best Student Paper Award) [10677-72]

1067721 Reconstruction enhancement of noisy data in terahertz pulse time-domain holography by iterative procedure [10677-73]

\section{ADVANCED METHODS V: HARSH ENVIRONMENTS}

1067722 Digital holography for erosion monitoring inside the ITER Tokamak (Invited Paper) [10677-74]

1067724 Digital holographic microscopy for remote life detection [10677-76]

1067725 Marine particles investigation by underwater digital holography [10677-77]

1067726 MEMS-based serial LiDAR detection and imaging architecture for automated surveillance of undersea marine life [10677-78]

\section{MODELLING, COMPUTATION AND DESIGN IV}

1067728 Supervised machine learning for 3D microscopy without manual annotation: application to spheroids [10677-80]

1067729 L1-norm minimization-based accurate diffraction field calculation method emitted by threedimensional objects [10677-85]

10677 2A An alternative method to correct translation positions in ptychography [10677-81] 
$106772 \mathrm{~B} \quad$ Single-frame fringe pattern analysis using modified variational image decomposition aided by the Hilbert transform for fast full-field quantitative phase imaging [10677-82]

$106772 \mathrm{D}$ Shape measurement by inverse raytracing [10677-84]

\section{POSTER SESSION}

$106772 \mathrm{E} \quad$ Recognition of blastic cells in human peripheral blood by diffraction phase microscopy [10677-87]

$106772 \mathrm{G}$ The multichannel pyrometer of the spectral ratio for on-line monitoring in the powder bed additive technologies [10677-89]

$106772 \mathrm{H} \quad$ Multiscale photoacoustic microscopy imaging with image improvement and quantification technique [10677-90]

1067721 LED misalignment determination in LED illumination optics using hole grid pattern distribution [10677-91]

$106772 \mathrm{~J} \quad$ Optimizing phase object reconstruction using an in-line digital holographic microscope and a reconstruction based on a Lorenz-Mie model [10677-92]

$106772 \mathrm{~K}$ Methods and challenges in laser-induced damage threshold evaluation of volumetric photopolymerized micro-structures [10677-93]

$106772 \mathrm{~L} \quad$ Broadband Sb/B4C multilayer mirrors for XUV spectroscopy applications [10677-94]

10677 2M Design and calibration for a Full-Stokes imaging polarimeter. [10677-96]

$106772 \mathrm{~N}$ Unconventional imaging with radial Walsh filters [10677-97]

1067720 Dynamic microparticle manipulation through light structures generated by a self-calibrated Liquid Crystal on Silicon display [10677-98]

10677 2P Image quality enhancement based on real-time deconvolution and super resolution [10677-99]

$106772 R \quad$ MWIR infrared gating imaging with uncooled PbSe FPAs for surveillance applications [10677-101]

1067725 Diffraction model of a plenoptic camera for in-situ space exploration [10677-102]

10677 2T Aberrations analysis of a Focused Plenoptic Camera [10677-103]

$106772 \mathrm{U}$ Comparison of reconstruction approaches for plenoptic imaging systems [10677-104]

$106772 \mathrm{~V}$ On the use of image quality measures of multi-views in light sheet fluorescence 3D microscopy [10677-105] 
$106772 X \quad$ Application of stacked sparse autoencoder in automated detection of glaucoma in fundus images [10677-107]

$106772 Y \quad$ Automated detection of glaucoma in fundus images using variational mode decomposition and textural features [10677-108]

$1067734 \quad$ Multispectral imaging technique for skin grafts' functional state assessment [10677-116]

1067736 Low-cost polarimetric imaging for surveillance [10677-118]

$1067737 \quad$ Applications of polarimetric imaging in the oil film detection [10677-119]

1067738 Database of polarimetric and multispectral images in the visible and NIR regions [10677-120]

1067739 Description of near-field digital in-line holography using vectorial Rayleigh-Sommerfeld integral [10677-121]

10677 3A Combined digital-DOE holographic interferometer for force identification in vibroacoustics [10677-122]

10677 3B Data acquisition from digital holograms of particles [10677-123]

10677 3C Quantitative phase imaging of low-cost digital in-line holography in comparison with off-axis digital holographic microscopy [10677-124]

$106773 \mathrm{E} \quad$ Investigation of the effect of the spectral and angular selectivities of three-dimensional color security holographic stereograms on the process of the reconstruction of 3D images [10677-126]

10677 3F Position measurement of in-line microbid holograms using an autoregressive method [10677-127]

10677 3G Photon-counted integral holography using orthographic projection images [10677-128]

$106773 \mathrm{H} \quad$ Optimization of the complex coherence function $\Gamma$ for diffraction-based wavefront transformations [10677-129]

$1067731 \quad$ Terahertz hyper-spectral imaging of lab-prepared versus commercial paracetamol tablets and potential applications [10677-130]

10677 3J Solution for reconstruction enhancement of pulsed broadband THz holograms recorded with small area detectors [10677-131]

10677 L Imaging of supersonically expanded and pulsed molecular beams by nonlinear photoionization processes [10677-112]

$106773 \mathrm{~N} \quad$ Multimodal fluorescence imaging navigation for surgical guidance of malignant tumors in photosensitized tissues of neural system and other organs [10677-86] 
$106773 Q \quad$ Investigation of a compressive line sensing hyperspectral imaging sensor [10677-11]

$106773 R \quad$ Methods of image correction formed on horizontal long paths [10677-65]

Proc. of SPIE Vol. 10677 1067701-9

Downloaded From: https://www.spiedigitallibrary.org/conference-proceedings-of-spie on 25 Apr 2023 Terms of Use: https://www.spiedigitallibrary.org/terms-of-use 
Proc. of SPIE Vol. 10677 1067701-10 Downloaded From: https://www.spiedigitallibrary.org/conference-proceedings-of-spie on 25 Apr 2023
Terms of Use: https://www.spiedigitallibrary.org/terms-of-use 


\section{Authors}

Numbers in the index correspond to the last two digits of the seven-digit citation identifier (CID) article numbering system used in Proceedings of SPIE. The first five digits reflect the volume number. Base 36 numbering is employed for the last two digits and indicates the order of articles within the volume. Numbers start with 00, 01, 02, 03, 04, 05, 06, 07, 08, 09, OA, OB...0Z, followed by 10-1Z, 20-2Z, etc.

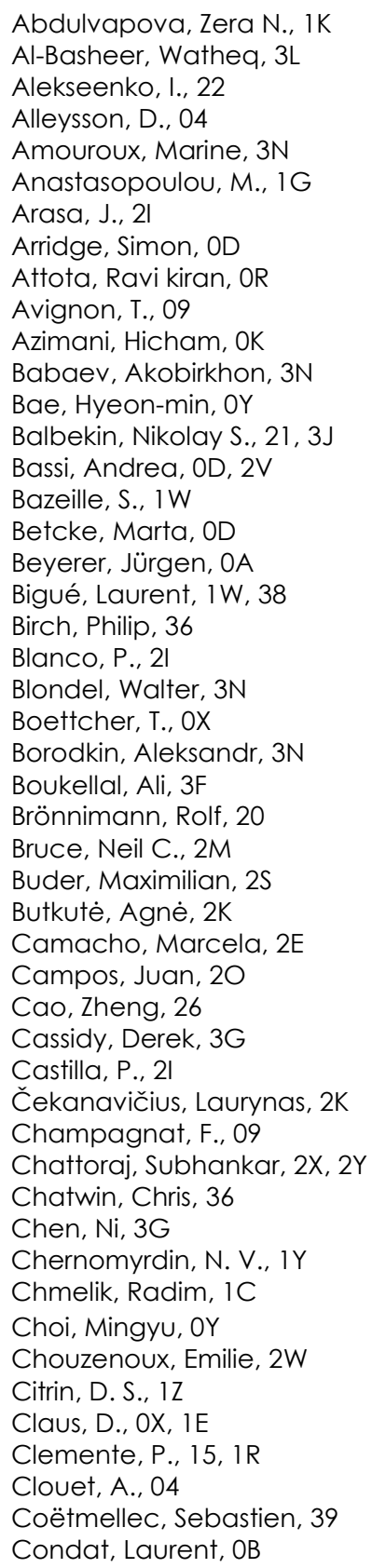

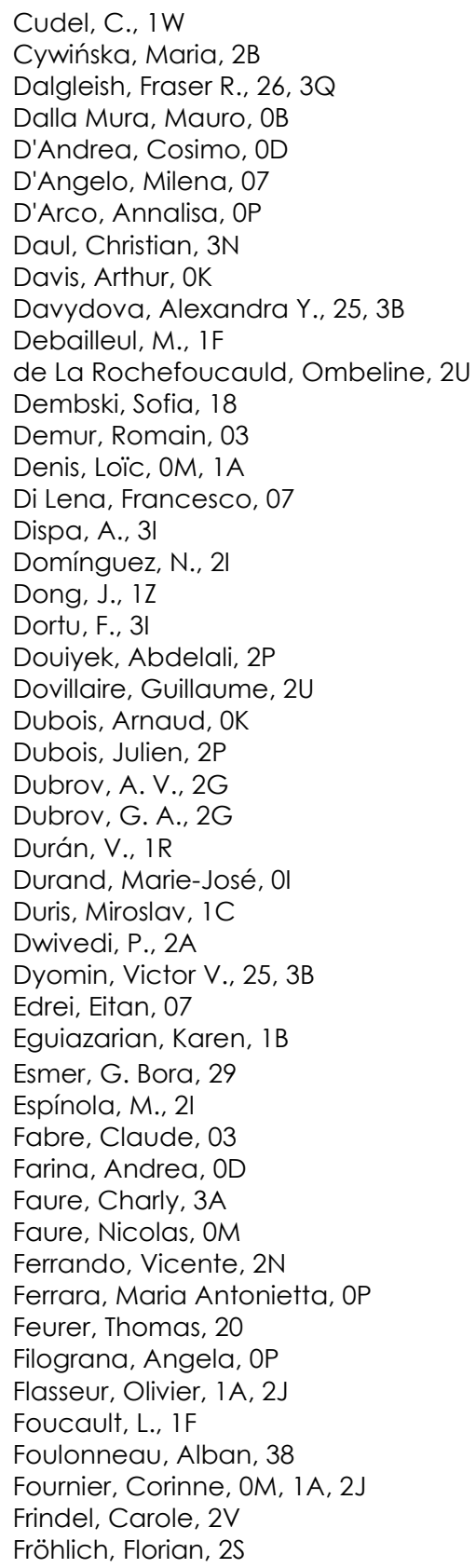


Furlan, Walter D., 2N

Fütterer, G., 3H

G., Hanu Phani Ram, 3C

Galstyan, Gagik R., 1K

Gao, Peng, 10

Garcia, C., 2I

Garcia-Caurel, Enric, 18

García-Díaz, Antón, 05, 2R

Garuccio, Augusto, 07

Gendre, Luc, IW, 38

Ginhac, Dominique, 2P

Glasenapp, Carsten, 2D

Glebov, V. N., 2 G

Golbin, Denis, 3N

Gonsalves, Robert A., is

Gorevoy, Alexey V., OF

Gorodetsky, Andrei A., 21, 3J

Gorpas, D., 1G

Grachev, Pavel V., 00, $1 \mathrm{~K}$

Granier, Xavier, $2 \mathrm{U}$

Grisard, Arnaud, 03

Groeber-Becker, Florian Kai, 18

Grosso, Alessandro, 2T

Gryadunova, A. A., $1 Y$

Gu, Feifei, 37

Guo, Jinxin, $1 X$

Hack, Erwin, 20

Haeberlé, Olivier, 0J, IF

Harms, Fabrice, $2 \mathrm{U}$

Hartrumpf, Matthias, OA

Healy, John J., 3G

Heggarty, Kevin, 3A

Hernandez, Y., 3I

Herzog, Charlotte, $2 \mathrm{U}$

Heyrman, Barthélémy, 2P

Hibst, R., OX

Huaman, Rosa, 28, 2V

Hubert, Ph., 3I

lemmi, Claudio, 20

Ignateva, I. V., 34

Jagannathan, G., 22

Jauregui-Sánchez, Y., 15

Jeon, Seungwan, $2 \mathrm{H}$

$\mathrm{Ji}$, Minsu, OY

John, Renu, 3C

Jolivet, Frédéric, OM, $1 \mathrm{~A}$

Jonušauskas, Linas, 2K

Karalkin, P. A., $1 Y$

Karasik, V. E., 1 Y

Katkovnik, Vladimir, 1B

Katyba, G. M., IY

Kempenaars, M., 22

Khokhlov, Demid D., OF

Kim, Chulhong, 2H

Kim, Jaemyoung, OY

Kim, Jin Young, $2 \mathrm{H}$

Klemm, U., 1G

Koch, M., $1 \mathrm{G}$

Kolesnikov, Alexei O., OE, $2 \mathrm{~L}$

Kolontaeva, G. S., IY
Kondratenko, Valeriy V., 2L

Konijnenberg, A. P., 2A

Kopylets, Igor A., 2L

Kopylov, E. A., 3R

Krutz, Ulrike, 2S

Kucheryavenko, A. S., IY

Kulya, Maksim S., 21, 3J

Lallier, Eric, 03

Lancis, J., 15, 1R

Längle, Thomas, OA

Lapray, Pierre-Jean, 38

Larobina, Michele, OP

Lavrinov, V. V., 3R

Le Besnerais, G., 09

Lebrun, Denis, 39

Lebrun, Mickael, 01

Lee, Haeil, OY

Lee, Hee Ryung, 18

Lefort, Claire, OV, 2W

Le Meur, Julien, 3A

Lenz, Armin J. M., 15

Levecq, Olivier, OK

Leveca, Xavier, $2 \mathrm{U}$

Leviandier, LUC, 03

Li, Guitao, 10

Li, Pengcheng, 18

Li, Yanjun, 26, 3Q

Liewer, K., 24

Lindensmith, C., 24

Lingenauber, Martin, $2 S$

Linkov, Kirill G., $1 \mathrm{~K}$

Liu, Jing, 37

Liu, Sunxiangyu, 10

Lizana, Angel, 20

Locquet, A., 12

Longo, Elena, $2 \mathrm{U}$

Loran, Oleg, 3N

Loschenov, Victor B., 1K, 34, 3N

Loshchenov, Maxim, 3N

Lotz, Christian, 18

Ludwig, Stephan, 17

Lukin, V. P., 3R

Lushnikov, D. S., 3E

Machado, Federico, 2N

Machikhin, Alexander S., OF

Makarov, V. I., 00, 34

Malinauskas, Mangirdas, $2 \mathrm{~K}$

Malutin, A. M., 2G

Marin, Yoan, $2 \mathrm{P}$

Markin, V. V., 3E

Márquez, Andrés, 20

Mazzilli, Aldo, 07

Méès, Loïc, OM, $2 \mathrm{~J}$

Mehta, Shalin B., OW

Melchert, Wolfgang, OA

Melis, M., $1 Z$

Méndez-Rial, Roi, 05, 2R

Meyer, Johannes, OA

Mico, Vicente, 2B

Mieog, J. S. D., IJ 
Mignard-Debise, Loïs, $2 U$

Minin, I. V., IY

Minin, O. V., IY

Mirzade, F. Kh., 2G

Mitéran, Johel, $2 \mathrm{P}$

Mokdad, Rabah, 3F

Momey, Fabien, 0M, 1A

Monroy Ramírez, Freddy, 2E

Monsoriu, Juan A., 2N

Montes González, Iván, 2M

Moreno, Ignacio, 20

Morvan, Loïc, 03

Muniraj, Inbarasan, $3 \mathrm{G}$

Nadeau, J., 24

Naumov, Alexander A., OF

Nguyen, D. T., 3l

Norkin, N. E., 1Y

Novikova, Tatiana, 18

Ntziachristos, V., $1 \mathrm{G}$

Odinokov, S. B., 3E

Olivier, Thomas, 2J

Olshukov, Alexey S., 3B

Organista-Castelblanco, C., 2E

Ossikovski, Razvigor, 18

Osten, Wolfgang, 0X, 17, 22

Ouyang, Bing, 26, 3Q

Parfenov, V. A., $1 Y$

Patorski, Krzysztof, 2B

Pedrini, Giancarlo, OX, 17, 1E, 22

Pepe, Francesco V., 07

Pereira, S. F., 2A

Perrot, Jean-LUC, OK

Pesquet, Jean-Christophe, $2 \mathrm{~W}$

Petrov, Nikolay V., 1B, 21, 3J

Pezerat, Charles, 3A

Pfeiffer, Pierre, 3F

$\mathrm{Pi}$, Francesc, 20

Picart, Pascal, 0I, 19, 3A

Picone, Daniele, OB

Pinston, Frédéric, $\mathrm{OM}$

Pisani, Marco, 06

Pizarro, C., 2

Poittevin, Julien, 3A

Polonschii, Cristina, $\mathrm{OL}$

Polovtsev, Igor G., 25

Pominova, D. V., 00, 34

Popescu, Gabriel, OL

Potapov, Aleksandr, 3N

Pratiher, Sawon, $2 X, 2 Y$

Principe, Jose, 26

Ragozin, Evgeny N., OE, $2 \mathrm{~L}$

Ramos, Brian, 26

Ranjan, Rajeev, OP

Rasti, Pejman, 28

Reshetov, I. V., 34

Riviere, Charlotte, 28, 2V

Rodin, P.S., 2G

Romanishkin, I. D., 00

Rong, LU, 1Q, IX

Rousseau, David, 28, 2V
Ryabova, A. V., 00, 34

Ryle, James P., $3 G$

Sabater, J., 09

Saidani, Nadia, 3F

Saveleva, T. A., 34

Scarcelli, Giuliano, 07

Scharf, Toralf, 2T

Selin, A. A., 3R

Serabyn, E., 24

Shaked, Natan T., $\mathrm{OH}$

Shatokhin, Alexey N., OE, 2L

Sheppard, Colin J. R., OW

Sheridan, John T., 3G

Shevkunov, Igor, 1B

Simon, B., $1 F$

Singh, Alok K., 17

Siret, David, OK

Sirleto, Luigi, OP

Smirnov, A. V., 3E

Smolyanskaya, O. A., IY

Soldevila, F., 1R

Song, Zhan, 37

Souto-López, Álvaro, 05, 2R

Steiner, Rudolf, 3N

Strbkova, Lenka, 1C

Strobl, Klaus H., $2 S$

Tajahuerce, E., 15, 1R

Taphanel, M., OX

Tawk, Teddy, 39

Thouand, Gérald, 01

Torres-Ruiz, Fabian A., 20

Treps, Nicolas, 03

Trinh, Dinh-Hoan, 3N

Trouvé-Peloux, P., 09

Trusiak, Maciej, 2B

Turpin, Alex, 20

Twardowski, Michael, $3 Q$

Urbach, H. P., 2A

Vahrmeijer, A. L., $1 \mathrm{~J}$

Vaillant, J., 04

Valente, Carmen, OP

Valentini, Gianluca, OD

Valzania, Lorenzo, 20

Van Eeckhout, Albert, 20

van Manen, L., $1 \mathrm{~J}$

Vargas, Asticio, 20

Vayakis, G., 22

Verrier, N., $1 \mathrm{~F}$

Vishnyakov, Eugene A., OE, $2 \mathrm{~L}$

Vishwakarma, Karan, 2X, 2Y

Vonmetz, Kurt, 2T

Vuorenkoski, Anni K., 26

Wallace, K., 24

Wan, Min, 3G

Wang, Dayong, 1Q, 1X

Wang, Fengpeng, 1Q

Wang, Yunxin, 1Q, 1X

Yin, Liuguo, 10

Yoo, Thomas Sang Hyuk, 18

Young, Rupert, 36 
Yu, Sungkwon, OY

Zavalov, Y. N., 2G

Zaytsev, K. I., IY

Zeitoun, Philippe, $2 \mathrm{U}$

Zhai, Changchao, $1 \mathrm{X}$

Zhang, Haolin, 20

Zhang, Xinyan, 10

Zhao, Jie, 1Q, $1 X$

Zhao, Juan, 37

Zhao, Kongya, 10

Zherdev, A. Y., 3E

Ziemons, E., 3I

Zolliker, Peter, 20

Zucco, Massimo, 06 


\title{
Conference Committee
}

\author{
Symposium Chairs
}

Francis Berghmans, Vrije Universiteit Brussel (Belgium)

Thierry Georges, Oxxius SA (France)

Harald Giessen, Universität Stuttgart (Germany)

Paul C. Montgomery, Université de Strasbourg (France)

Conference Chairs

Corinne Fournier, Université Jean Monnet Saint-Etienne (France)

Marc P. Georges, Université de Liège (Belgium)

Gabriel Popescu, University of Illinois at Urbana-Champaign

(United States)

Conference Programme Committee

Arun Anand, The Maharaja Sayajirao University of Baroda (India) Matthieu Boffety, Institut d'Optique Graduate School (France)

Pierre H. Chavel, Institut d'Optique Graduate School (France)

Nicholas Devaney, National University of Ireland, Galway (Ireland)

Julien Fade, Université de Rennes 1 (France)

Mathias Fink, Institut Langevin, ESPCI Paris (France)

Sylvain Gioux, ICube (France)

François Goudail, Institut d'Optique Graduate School (France)

Olivier Haeberlé, Université de Haute Alsace (France)

Giancarlo Pedrini, Institut für Technische Optik (Germany)

François Ramaz, Institut Langevin (France)

Neus Sabater, Technicolor (France)

Frans Snik, Leiden Observatory (Netherlands)

Enrique Tajahuerce, Universitat Jaume I (Spain)

Pauline Trouvé, ONERA (France)

\section{Session Chairs}

1 Modelling, Computation and Design I: Co-design for Unconventional Imaging

Pauline Trouvé, ONERA (France)

2 Modelling, Computation and Design II: Co-design for Unconventional Imaging

Corinne Fournier, Université Jean Monnet Saint-Etienne (France) 
Applications I: Bioimaging

Gabriel Popescu, University of Illinois at Urbana-Champaign (United States)

4 Applications II: Bioimaging

Olivier Haeberlé, Université de Haute Alsace (France)

5 Applications III: Micro- Nanoscopic Imaging

Philipp Kukura, University of Oxford (United Kingdom)

6 Modelling, Computation and Design III

Gabriel Popescu, University of Illinois at Urbana-Champaign (United States)

7 Advanced Methods I: Light Scattering

Enrique Tajahuerce, Universitat Jaume I (Spain)

8 Advanced Methods II: Quantitative Phase Imaging

Giancarlo Pedrini, Institut für Technische Optik (Germany)

JS1 Optics in Surgery I: Joint Session between Conferences 10677 and 10685

Sylvain Gioux, ICube (France)

JS2 Optics in Surgery II: Joint Session between Conferences 10677 and 10685

Dimitris Gorpas, Hwlmholz Zentrum München

9 Applications IV: Bioimaging

Gabriel Popescu, University of Illinois at Urbana-Champaign (United States)

10 Advanced Methods III: Quantitative Phase Imaging Eugen Gheorghiu, The International Center of Biodynamics (Romania)

11 Applications V: Wavefront Shaping and Restoration

Pierre H. Chavel, Institut d'Optique Graduate School (France)

12 Advanced Methods IV: Terahertz Imaging Marc P. Georges, Université de Liège (Belgium)

13 Advanced Methods V: Harsh Environments Marc P. Georges, Université de Liège (Belgium)

14 Modelling, Computation and Design IV

Corinne Fournier, Université Jean Monnet Saint-Etienne (France) 